Competence matters! Understanding biculturalism in ethnically diverse adolescents.

\author{
Alison E. F. Benbow \\ Institute of Psychology \\ FernUniversität in Hagen, Germany \\ \& \\ Adam Rutland \\ Department of Psychology \\ Goldsmiths, University of London, UK
}

This research was undertaken while both authors worked at the Department of Psychology, University of Kent, Canterbury, UK

To be considered for the Special Issue „Growing up with diversity“ in the Journal of Community and Applied Social Psychology

Word Count: 2190 (including Abstract, Keywords, References and Tables)

Corresponding author: Alison E. F. Benbow, Department of Psychology, FernUniversität in Hagen, Universitätsstr. 33, 58084 Hagen, Germany; e-mail: alison.benbow@fernunihagen.de; Tel.: +44 (0)15116807748. 


\begin{abstract}
The importance of bicultural competence in negotiating diversity was explored longitudinally with 228 bicultural British females. Bicultural competence predicted social-psychological adaptation and intergroup attitudes. It also mediated the effects of ethnic identity and perceived similarity on acculturation preferences. Implications for research on adolescents growing up with diversity are considered.
\end{abstract}

Keywords: bicultural competence, negotiating diversity, adolescence, ethnic identity, acculturation, minority youth 


\section{Competence matters! Understanding biculturalism in ethnically diverse adolescents.}

Bicultural children and adolescents - second or higher generation migrants or mixedethnicity individuals - are of specific interest when considering the impact of diversity on development, because diversity is much more than an experience to them: it is an integral part of their selves (e.g., Verkuyten \& Pouliasi, 2002). From an early age, bicultural individuals negotiate their ethnic identity formation alongside a growing consciousness of belonging to more than one sociocultural group. Unlike other (monocultural) individuals, their whole development is therefore intrinsically linked with acquiring bicultural competence (BCC) relevant to their ethnic group(s) and society as a whole (Oppedal, 2006).

Research has shown that bicultural identification, or integration, is the most successful acculturation strategy for immigrants and their offspring in terms of psychological development (e.g., Berry, Phinney, Sam, \& Vedder, 2006) yet bicultural identity achievement is typically delayed and only occurs in late adolescence or adulthood (e.g. Phinney \& Alipuria, 2006). It is therefore important for social and developmental psychologists to understand the emergence of BCC during adolescence and how its promotion can support psychological well-being and societal cohesion in diverse social contexts.

Nonetheless, there is very little work that expressly considers bicultural competence in adolescence. BCC refers to a youth's ability to function effectively in multi-ethnic, pluralistic environments by displaying an understanding of the varying social norms of, and observable behavioural flexibility with regard to, at least two cultural groups (Rotheram-Borus, 1993). Importantly, BCC does not necessitate cultural identification. In an extensive literature review, LaFromboise, Coleman and Gerton (1993) concluded that successful achievement of bicultural identification and adaptation may depend on an individual first gaining BCC. Such competence includes awareness and knowledge of the history, institutions, rituals and everyday practices of a given culture (knowledge), the belief or confidence that one can live effectively within two groups without compromising one’s sense of cultural identity 
(bicultural efficacy), the ability to communicate ideas and feelings to members of a given culture and to develop culturally appropriate behaviours or roles (role repertoire), and having a well-developed support system (social support).

Similar individual dimensions relevant to identification and acculturation have been discussed (e.g., Phinney \& Alipuria, 2006), but there is no published, empirical research that considers a detailed model of bicultural competence. Little is known about how BCC connects to other developmental and inter-group processes relevant to children growing up with diversity. Therefore, this longitudinal study explored whether dimensions of BCC predict children's social-psychological adaptation (their self-esteem and peer acceptance) and their positive inter-group attitudes (the desire to engage with ethnic groups other than their own) over time. We also considered the importance of BCC for achieving bicultural identification or integration. In the acculturation literature integration is often conceptualised as a desire for cultural maintenance paired with a desire for out-group contact. Past research has shown that ethnic identification is predictive of the former (e.g., Berry et al., 2006), while perceived similarity may aid the latter (e.g., Stathi, Cameron, Hartley, \& Bradford, 2014). Based on the outlined theoretical rationale, we would expect these known relationships to be influenced by an individual's BCC. We therefore tested our BCC dimensions as mediators of the relationship between ethnic identification and the desire for culture maintenance on the one hand and the relationship between perceived similarity and desire for out-group contact on the other hand.

\section{Method}

\section{Participants, Measures and Procedure}

228 bicultural female participants (aged 11 to $17, M=13.08, S D=1.47$, with a time of stay in the UK from 9 to 17 years, $M=12.87, S D=1.56$ at Time 1 ) living in London, UK, completed a questionnaire in group sessions during lesson time at two measurement points 
(six months apart). At both measurement times participants received an introduction and scale practice, then completed demographic information and the following measures: 1.) Bicultural competence (see Appendix) including cultural knowledge (British and ethnic), bicultural efficacy, role repertoire and social support. 2.) Preferences for immigrant culture maintenance and contact (Zagefka \& Brown, 2002). 3.) Phinney’s (1992) multigroup ethnic identity measure and 4.) Other group orientation scale. 5.) Perceived similarity between own and other groups (Piontkowski et al., 2001). 6.) The Rosenberg self-esteem scale (1965) and 7.) Peer group acceptance (Asher \& Wheeler, 1985). Scale properties are provided in Table 1.

\section{Results}

\section{Social-psychological and intergroup consequences of bicultural competence.}

Figure 1 depicts a structural equation model testing BCC at Time 1 (modeled as a single latent factor underlying cultural knowledge, bicultural efficacy, role repertoire, and social support) as a predictor of peer-group acceptance and self-esteem (as markers of socio-psychological adaptation) and other-group orientation at Time 2. This model, which indicated significant positive relationships between BCC and the three outcomes, fits the data well, $\chi^{2}(16)=$ 23.08, $p=.11 ; C F I=.95 ; \mathrm{TLI}=.92 ; R M S E A=.04$.

Bicultural competence and acculturation preferences. We simultaneously investigated the mediating effects of our five BCC dimensions on a) the relationship between ethnic identification and cultural maintenance preferences and b) the relationship between perceived similarity and desire for outgroup contact using bootstrap analyses with 50,000 samples to test the significance of indirect effects in Hayes' (2013) PROCESS macro for SPSS. The first mediation model (Figure 2) accounted for $9 \%$ of the criterion variance, $R^{2}=$ $.09, F(6,202)=3.50, p=.003$. The original direct effect of ethnic identification $(b=.22,95 \%$ CI $[.06, .39])$ was reduced to non-significance $(b=.01,95 \%$ CI $[-.20,-.22])$, while the total indirect effect of BCC on the relationship between ethnic identification and culture 
maintenance was significant $(b=.21,95 \%$ CI $[.05, .39])$ and was based on the specific indirect effect via ethnic knowledge ( $b=.21,95 \%$ CI $[.05, .39])$.

The second mediation model (Figure 3) accounted for $14 \%$ of the criterion variance, $R^{2}=.14, F(6,212)=5.79, p<.001$. The direct effect of perceived similarity $(b=.21,95 \% \mathrm{CI}$ $[.04, .38])$ was reduced to non-significance $(b=.16,95 \%$ CI $[-.01, .33])$. The total indirect effect of BCC on the relationship between perceived similarity and contact was nonsignificant ( $b=.05,95 \%$ CI [-.04, .11]), but there were small and significant specific indirect effects via British knowledge ( $b=.06,95 \%$ CI $[.01, .12]$ ), bicultural efficacy $(b=.03,95 \%$ CI [.003, .07]), and role repertoire $(b=-.03,95 \%$ CI [-.07, -.001]).

\section{Discussion}

Given the limited research in this area, our findings provide a genuinely explorative insight into consequences and role of bicultural competence in adolescents growing up in a diverse context. Nonetheless, our findings do complement and extend current research and theorizing. Specifically, we uncovered a well-fitting model where ethnic and British knowledge, bicultural efficacy, role repertoire and social support underlie a latent BCC factor that positively predicted social-psychological adaptation and other-group orientation. This supports the assertion that gaining BCC along several dimensions is beneficial to minority youth, because it facilitates adaptation and positive inter-group attitudes.

Our mediational analyses suggest that BCC is indeed an important precursor of an integration preference (and, by extension, bicultural identification). The full mediation of the relationship between ethnic identification and culture maintenance by ethnic knowledge highlights the close relationship of knowledge and identification in early adolescence and is in line with research on ethnic identity formation at this age (e.g., Phinney \& Alipuria, 2006). This finding suggests that BCC may be a particularly meaningful and informative concept in early adolescence before individuals develop deeper insights of ethnic identification. Our finding that knowledge about Britain, bicultural efficacy, and role repertoire mediated the 
effect of perceived similarity on contact preferences also showed that BCC is relevant when examining intergroup relations in early adolescence. Together these analyses highlight the potential of BCC in helping young adolescents successfully negotiate the diversity they are faced with in their daily lives.

Given the exploratory nature of this study and its limitations, such as the single sex sample and reliance on self-report measures, we do not want to overstate the generalizability of our findings or their interpretation. Nonetheless, we do believe that our findings indicate that BCC is a construct that merits further consideration especially in early adolescence. This interest is not just limited to bicultural minorities, who are negotiating diversity in their surroundings and within themselves. It is likely that majority children also have the opportunity to develop (bi-)cultural competence, especially where they find themselves in multicultural contexts. In light of the benefits of BCC for social-psychological adaptation and intergroup attitudes suggested in our analyses, one might even posit that all children would equally benefit from acquiring BCC in culturally diverse societies. Future research should also consider the impact of parents, educators and peers in transmitting and fostering cultural competence. Understanding more about when and how cultural competence matters would add to the emerging field and complement our knowledge of the psychological, situational and societal factors that improve social cohesion and integration in diverse communities. 


\section{References}

Asher, S. R. \& Wheeler, V. A. (1985). Children's Loneliness: A comparison of rejected and neglected peer status. Journal of Counseling and Clinical Psychology, 53 (4), 500505. doi:10.1037/0022-006X.53.4.500

Berry, J. W., Phinney, J. S., Sam, D. L. \& Vedder, P. (2006). Immigrant youth in cultural transition: Acculturation, identity and adaptation across national contexts. Mahwah, NJ: Lawrence Erlbaum Associates.

Hayes, A. F. (2013). Introduction to mediation, moderation and conditional process analysis: a regression based approach. New York: The Guilford Press.

LaFromboise, T., Coleman, H. \& Gerton, J. (1993). Psychological impact of biculturalism: Evidence and theory. Psychological Bulletin, 114, 395-412. doi:10.1037/00332909.114.3.395

Oppedal, B. (2006). Development and acculturation. In D. L. Sam \& J. W. Berry (Eds.), The Cambridge handbook of acculturation psychology (pp. 97-112). Cambridge: Cambridge University Press. doi:10.1017/CBO9780511489891.010

Phinney, J.S. (1992). The multigroup ethnic identity measure: A new scale for use with diverse groups. Journal of Adolescent Research, 7, 156-176. doi:10.1177/074355489272003

Phinney, J. S. \& Alipuria, L. L. (2006). Multiple social categorization and identity among multiracial, multiethnic, and multicultural individuals. Processes and implications. In R. J. Crisp and M. Hewstone (Eds.), Multiple Social Categorization: Processes, Models and Applications (Chapter 10). Hove: Psychology Press.

Piontkowski, U., Florack, A., Hoelker, P. \& Obdrzalek, P. (2000). Predicting acculturation attitudes of dominant and nondominant groups. International Journal of Intercultural Relations, 24, 1-26. doi:10.1016/S0147-1767(99)00020-6 
Rosenberg, M. (1965). Society and the adolescent self-image. Princeton, NJ: Princeton University Press.

Rotheram-Borus, M. J. (1993). Biculturalism among adolescents. In M. E. Bernal \& G. P. Knight (Eds.), Ethnic Identity: Formation and transmission among Hispanics and other ethnic minorities, 81-102. Albany: State University of New York Press.

Stathi, S., Cameron, L., Hartley, B., \& Bradford, S. (2014). Imagined contact as a prejudicereduction intervention in schools: the underlying role of similarity and attitudes. Journal of Applied Social Psychology, 44, 536-546. doi: 10.1111/jasp.12245

Verkuyten, M. \& Pouliasi, K. (2002). Biculturalism among older children: Cultural Frame Switching, Attributions, Self-Identification and Attitudes. Journal of Cross-Cultural Psychology, 33, 596-609. doi:10.1177/0022022102238271

Zagefka, H. \& Brown, R. (2002). The relationship between acculturation strategies, relative fit and inter-group relations: immigrant-majority relations in Germany. European Journal of Social Psychology, 32, 171-188. doi:10.1002/ejsp.73 
Table 1.

Scale properties and correlations between scales.

\begin{tabular}{|c|c|c|c|c|c|c|c|c|c|c|c|c|c|c|c|c|}
\hline & \multirow{2}{*}{$\begin{array}{r}\text { Time } 1 \\
M(S D)\end{array}$} & \multicolumn{3}{|c|}{ Time 2} & \multicolumn{12}{|c|}{ Correlations } \\
\hline & & $\alpha$ & $M(S D)$ & $\alpha$ & 1 & 2 & 3 & 4 & 5 & 6 & 7 & 8 & 9 & 10 & 11 & 12 \\
\hline 1. Ethnic Knowledge & $5.34(1.12)$ & .88 & $5.49(1.14)$ & .90 & $.63^{*}$ & $.23^{*}$ & -.07 & $.14^{*}$ & $.33 *$ & $.25 *$ & .02 & $.65 *$ & $.14^{*}$ & .08 & $.18^{*}$ & $.17 *$ \\
\hline 2. British Knowledge & $5.35(1.04)$ & .85 & $5.54(1.07)$ & .90 & $.24^{*}$ & $.67 *$ & $.21 *$ & $.30 *$ & $.20^{*}$ & .04 & $.27^{*}$ & .10 & $.17 *$ & $.40^{*}$ & $.29 *$ & $.19 *$ \\
\hline 3. Bicultural Efficacy & $4.82(1.07)$ & .57 & 4.99 (1.13) & .60 & .04 & $.20^{*}$ & $.51 *$ & $.41^{*}$ & $.18^{*}$ & .09 & .13 & -.09 & $.23^{*}$ & $.14^{*}$ & .13 & .13 \\
\hline 4. Role Repertoire & $5.43(0.92)$ & .69 & $5.58(0.87)$ & .64 & $.16^{*}$ & $.27 *$ & $.41^{*}$ & $.50 *$ & $.32 *$ & .04 & -.01 & .04 & $.26 *$ & $.15^{*}$ & $.26^{*}$ & $.17 *$ \\
\hline 5. Social Support & $5.30(1.13)$ & .76 & $5.38(1.14)$ & .82 & $.24 *$ & $.25^{*}$ & .11 & $.28 *$ & $.51 *$ & $.13 *$ & -.04 & $.36^{*}$ & .12 & $.16^{*}$ & $.46^{*}$ & $.38 *$ \\
\hline 6. Culture Maintenance & $4.74(1.22)$ & .78 & $4.62(1.31)$ & .81 & $.30^{*}$ & .03 & .10 & .13 & .01 & $.43 *$ & $.18^{*}$ & $.32 *$ & $.20 *$ & .01 & $.18^{*}$ & .08 \\
\hline 7. Contact & $4.68(1.22)$ & .65 & $4.73(1.32)$ & .70 & $.14^{*}$ & $.26^{*}$ & $.17^{*}$ & .08 & -.07 & $.25 *$ & $.49 *$ & .03 & .08 & $.28 *$ & $.17^{*}$ & .12 \\
\hline 8. Ethnic Identity & $4.84(1.08)$ & .90 & $4.89(1.05)$ & .90 & $.64^{*}$ & .07 & .01 & .05 & $.20 *$ & $.20 *$ & .13 & $.74 *$ & $.21 *$ & -.05 & $.14^{*}$ & $.14^{*}$ \\
\hline 9. Other Group Orientation & $5.59(0.98)$ & .71 & $5.65(0.99)$ & .72 & $.21^{*}$ & $.25^{*}$ & $.27 *$ & $.45^{*}$ & $.18^{*}$ & .07 & $.15^{*}$ & $.21^{*}$ & $.35 *$ & .10 & $.21^{*}$ & $.20^{*}$ \\
\hline 10. Perceived Similarity & $4.18(1.05)$ & .78 & $4.36(1.23)$ & .86 & .06 & $.33^{*}$ & $.14^{*}$ & $.18^{*}$ & $.15^{*}$ & .02 & $.21 * *$ & .01 & $.14^{*}$ & $.47 *$ & $.16^{*}$ & .10 \\
\hline 11. Self-Esteem & $5.17(1.02)$ & .83 & $5.22(1.09)$ & .87 & $.26^{*}$ & $.20^{*}$ & $.13^{*}$ & $.27 *$ & $.39 *$ & .12 & .04 & $.22 *$ & $.22 *$ & .05 & $.71^{*}$ & $.49 *$ \\
\hline 12. Peer Acceptance & $5.96(1.05)$ & .83 & $6.05(0.89)$ & .78 & $.17^{*}$ & $.20^{*}$ & .04 & .11 & $.31^{*}$ & .02 & .04 & $.14^{*}$ & .12 & .04 & $.38^{*}$ & $.62 *$ \\
\hline
\end{tabular}

Note. All scales were presented on 7 points from 1 (strongly disagree) to 7 (strongly agree) and are coded so that high values indicate high agreement to the construct. Autocorrelations (Pearson's $r$ ) are presented in bold on the diagonal (Time 1 below/Time 2 above the diagonal). $* p<.05$ 


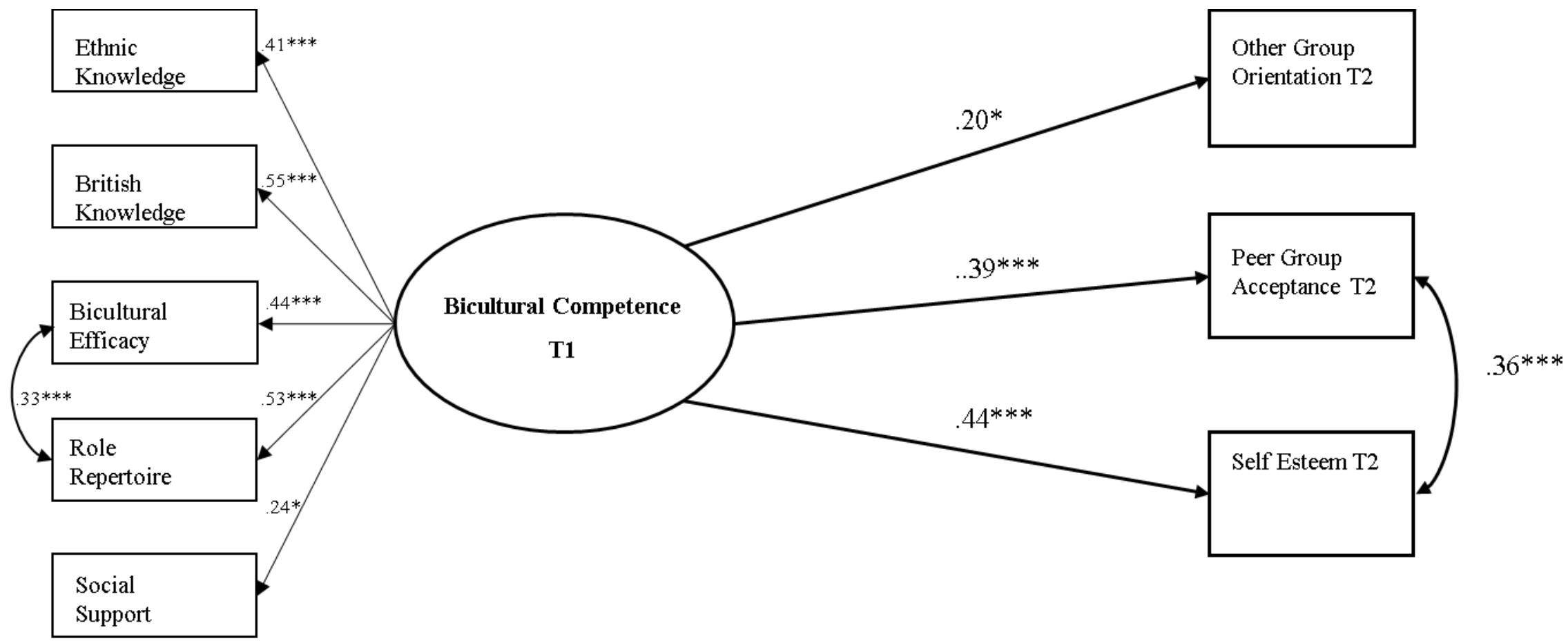

Figure 1. Prediction of social and psychological adjustment (other group orientation, peer group acceptance and self-esteem) at Time 2 from bicultural competence at Time 1 .

${ }^{*} p<.05 .{ }^{* *} p<.01$. ${ }^{* * *} p<.001$. 


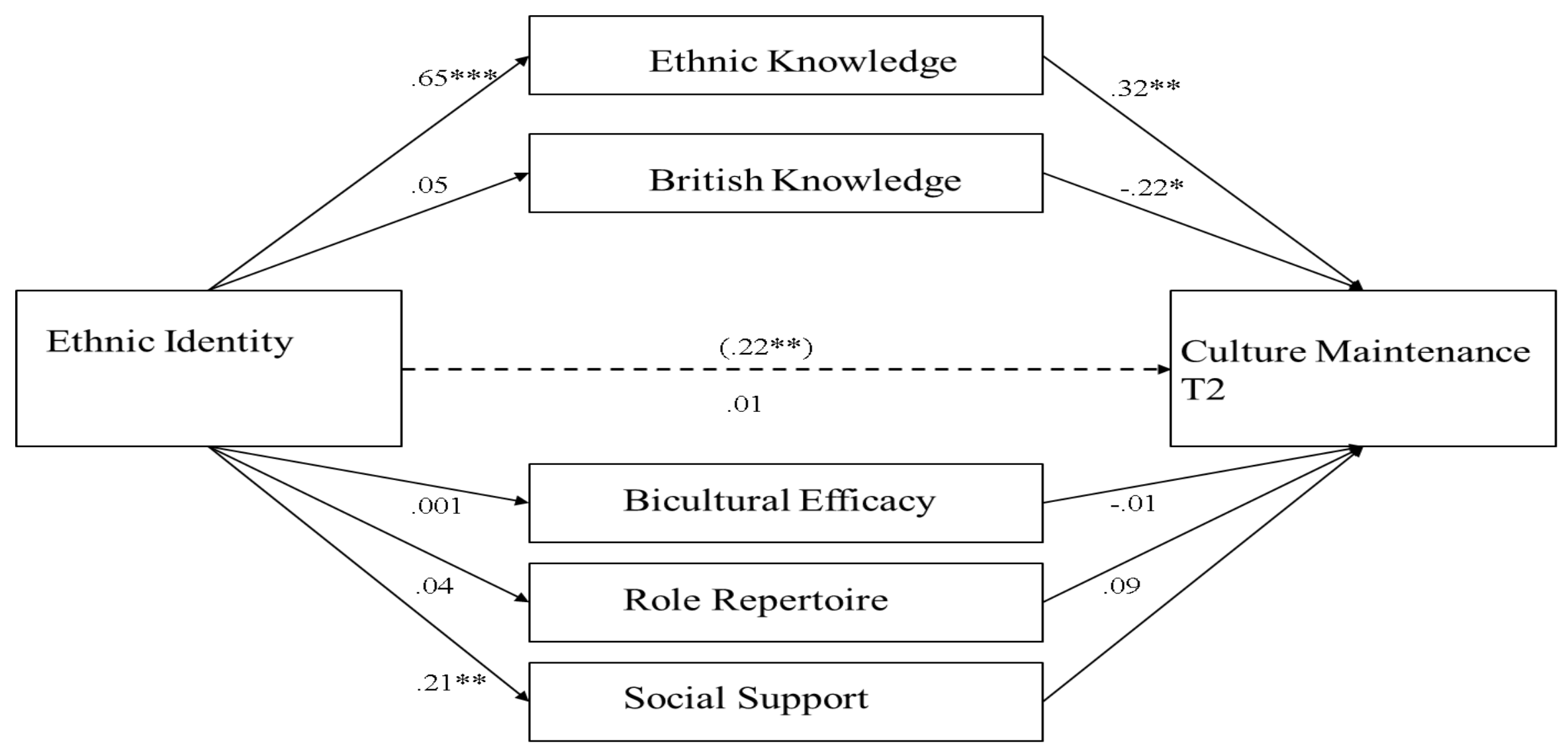

Figure 2. Multiple mediation model of the relationship between ethnic identity (Time 1) and culture maintenance (Time 2) mediated by facets of bicultural competence (Time 1). Bootstrapped standardized regression coefficients (b) are provided (unmediated effect in parentheses)

${ }^{*} p<.05 . *^{*} p<.01 . *^{* *} p<.001$. 


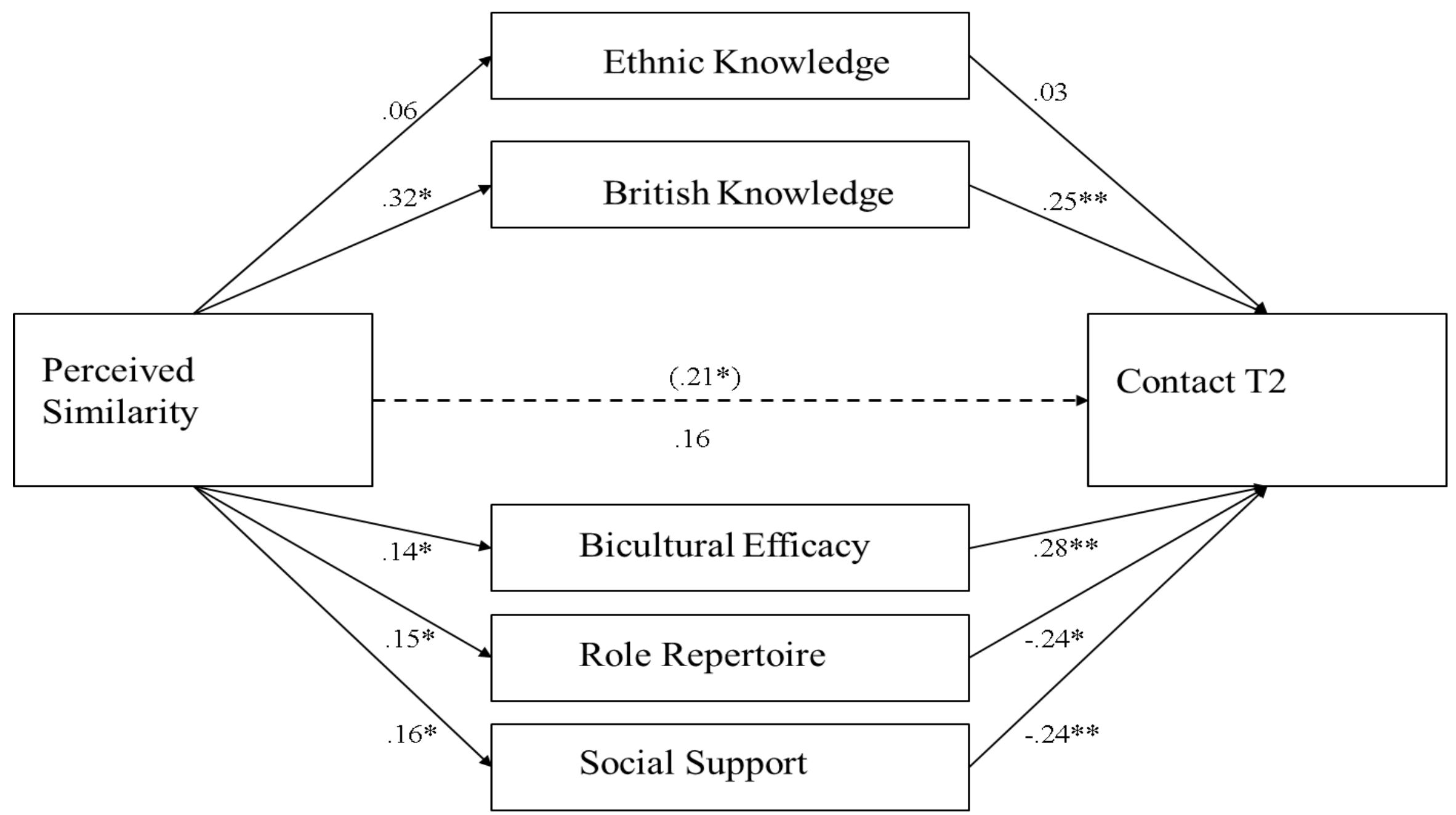

Figure 3. Multiple mediation model of the relationship between perceived similarity (Time 1) and preferences for contact (Time 2) mediated by facets of bicultural competence (Time 1). Bootstrapped standardized regression coefficients (b) are provided (unmediated effect in parentheses).

${ }^{*} p<.05 .{ }^{* *} p<.01 .{ }^{* * *} p<.001$. 


\section{Appendix 1}

\section{Bicultural Competence Items}

\section{Ethnic Knowledge}

The next section covers various questions that ask for the strength of your knowledge about aspects of your ethnic group. Please indicate your answer by circling the number that reflects your knowledge best. In this case “your ethnic group" refers to the general group you and your parents belong to rather than your individual ethnic identity.

$1=$ very poor, $2=$ poor, $3=$ somewhat poor, $4=$ neutral, $5=$ somewhat $\operatorname{good}, 6=\operatorname{good}, 7=\operatorname{very} \operatorname{good}$

My grasp of the traditions of my ethnic group is...

My comprehension of the important institutions (e.g. religious, political and social) of my ethnic group is... The understanding I have of my ethnic group's customs is...

The knowledge I have of my ethnic group's food is...

The comprehension I have of my ethnic group's history is...

My familiarity with the music of my ethnic group is...

My knowledge of the values that my ethnic group supports is...

\section{British Knowledge}

The next section covers various questions that ask for the strength of your knowledge about certain aspects of Britain. Please indicate your answer by circling the number that reflects your level of knowledge best.

$1=$ very poor, $2=$ poor, $3=$ somewhat poor, $4=$ neutral, $5=$ somewhat good, $6=\operatorname{good}, 7=\operatorname{very}$ good

My grasp of British history is...

My knowledge about the important (e.g. religious, political and social) institutions in Britain is...

My familiarity with British music is...

The knowledge I have about British food is...

My understanding of British values is...

The knowledge I have of British customs is...

My understanding of British traditions is...

\section{All others}

The following section covers various questions that ask for the strength of your agreement to a statement. Please indicate your answer by circling the number that reflects your opinion best.

1= strongly disagree, $2=$ disagree, $3=$ disagree somewhat, $4=$ neutral, $5=$ agree somewhat, $6=$ agree, $7=$ strongly agree

Where appropriate "your ethnic group or ethnicity" refers to the general group you and your parents belong to rather than your individual ethnic identity. Wherever culture(s) are not specified please consider the impact of ALL cultures that have an influence on your life (e.g. possible culture(s) of origin, as well as British culture).

\section{Role Repertoire}

I can relate to people from different cultures.

I only feel familiar dealing with my own culture. (reversed)

I have no problem relating to people from different cultures.

I do not find it easy to interact with people from different cultures. (reversed)

I believe I can fit in with all cultural groups.

I empathize with other peoples cultural experiences.

\section{Bicultural Efficacy}

I think it is possible for me to participate in two or more cultures.

Adapting to more than one cultural group is not an effective way to live my life. (reversed)

It is necessary to decide on one culture that is most important in my life. (reversed)

It is desirable to achieve a bi- (or multi-) cultural identity.

\section{Social Support}

When I need suggestions on how to deal with a personal problem, I know someone I can turn to.

I feel that there is no one I can share my most private worries and fears with. (reversed)

There are many people I can talk to about the issues surrounding my cultural beliefs and values.

There is someone I can turn to for advice about handling problems with my family.

There aren't many people who help me resolve cultural conflict. (reversed) 


\section{Biculturalism in ethnically diverse youth}

15

In a family crisis it would be difficult to find someone who could give me good advice about how to handle it. (reversed) 